
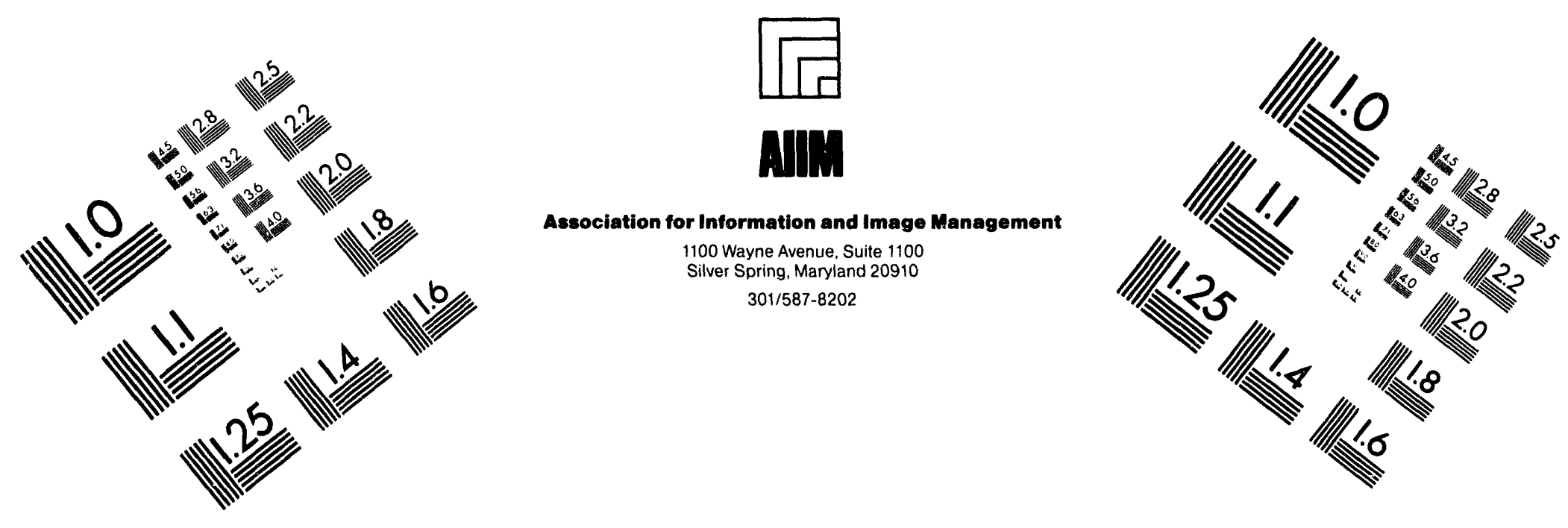

\title{
Centimeter
}

$\begin{array}{llllllllllllllll}1 & 2 & 3 & 4 & 5 & 6 & 7 & 8 & 9 & 10 & 11 & 12 & 13 & 14 & 15 & \mathrm{~mm}\end{array}$

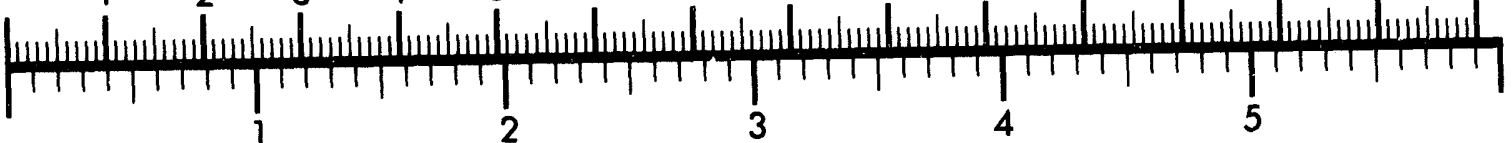
Inches

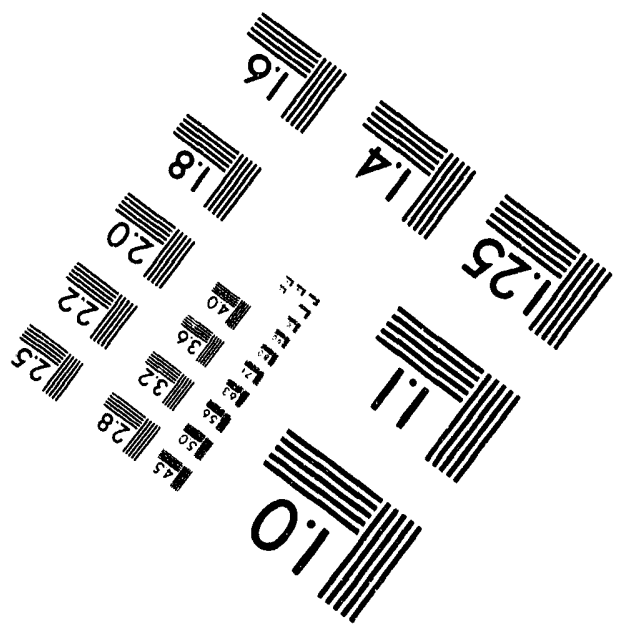

MANUFACTURED TO AIIM STANDARDS

BY APPLIED IMAGE, INC.

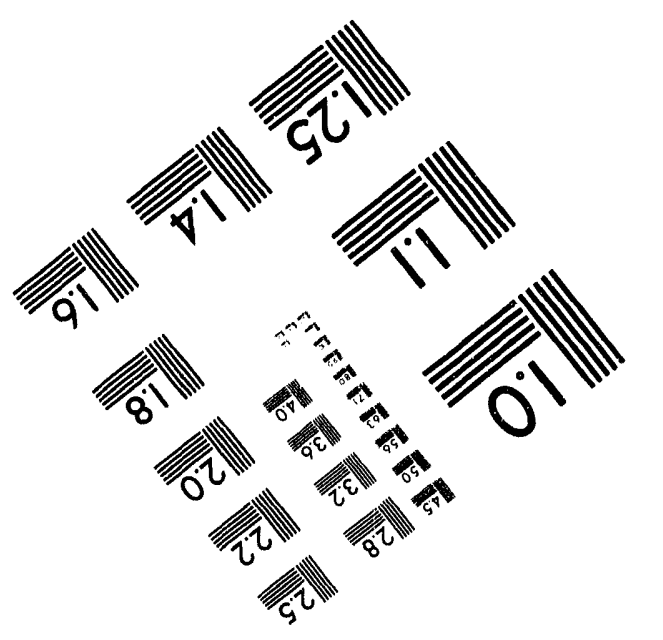



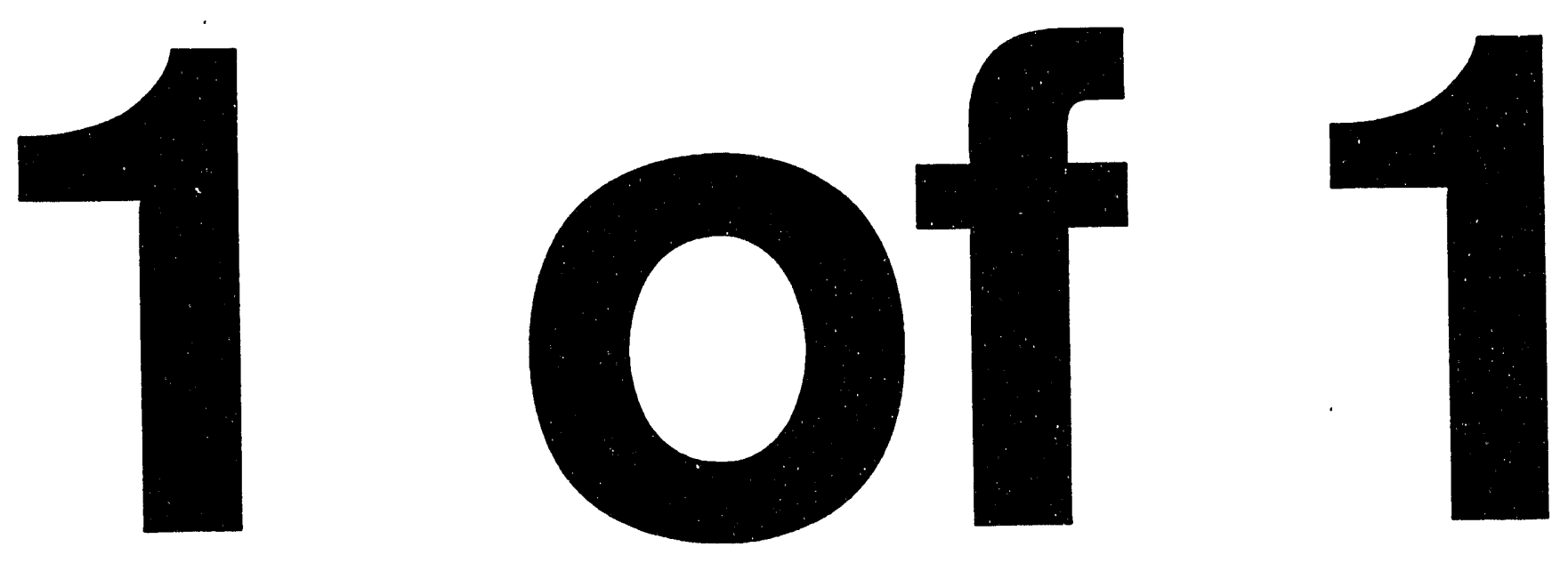


$$
\text { DOE ER/ }|382|--T \mid
$$

FINAL REPORT

\title{
COMPLEX TEMPORAL AND SPATIAL PATTERNS
}

\section{IN NONEQUILIBRIUM PROCESSES}

\author{
DOE Grant DE-FG05-88-13821
}

December 1, 1987 - November 30, 1992

We have used dynamical systems methods to study and characterize bifurcations and pattern formation in a variety of nonequilibrium systems. Some accomplishments during the current grant period include the following:

- development of information-theoretic methods for analyzing chaos

- development of new types of chemical reactors for the study of sustained reaction-diffusion patterns (using gels to suppress convection)

- development of a reactor that exploits pattern formation to extract short-lived intermediate species from a chemical reaction

- observation of a blfurcation from periodic to quasiperiodic rotating chemical spiral patterns

- first observation of a Turing blfurcation: a transition from a spatially uniform state to a stationary chemical pattern

- development of a method for extracting noise strength in ramped convection experiments

- discovery of self-similar fractal structure of zinc clusters formed in electrodeposition

- discovery of a dynamical instability in the propagation of cracks

In the following we describe our work on dynamical systems [1-11], chemical oscillations and chaos [12-20], chemical spatial patterns [21-39], instabilities in fluid dynamics [40-45], electrodeposition clusters [46-49], the ballast resistor [50], and crack propagation [51-52]. The reference numbers are to the publications on the DOEsupported research (10 papers in The Physical Review, 7 in Physica D, 6 in Journal of Chemical Physics, 4 in Physical Review Letters (plus two PRL "Comments"), 4 in Journal of Physical Chemistry, 2 in Nature, 1 each in several other refereed journals, and 6 in conference proceedings volumes). References 53-70 are to other work and are given at the end of the Final Report.

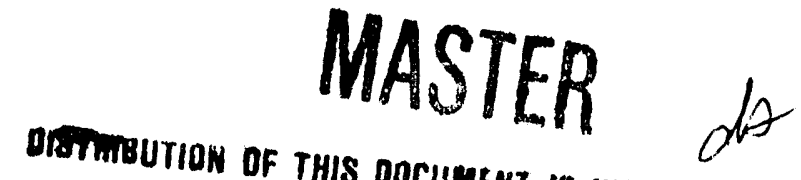

Derminution of THIS DOCuMENT is UNLIMITED 
Final Report--page 2

\section{DYNAMICAL SYSTEMS}

\section{Information theory}

The quantity mutual information, first introduced by C. E. Shannon in the $1940 \mathrm{~s}$, and its generalization redundancy have been examined for chaotic time series data and found to provide criteria for the choice of parameters in the construction of phase space attractors [1]. It was clear from the earliest analyses of attractors that dynamical invariants such as Lyapunov exponents are difficult to extract if the time delay used in constructing a strange attractor from time series data is too short or too long, but no criterion existed for the optimum delay. Our analysis shows that the optimum delay time corresponds to a minimum in the mutual information function. The analysis of the redundancy also yields the entropy and the measurement accuracy.

Information theoretic techniques for extracting parameters for phase portrait construction are superior to techniques based on autocorrelation functions or singular value decomposition; the redundancy analysis arises from a general notion of independence, while singular systems analysis is based on linear independence [2].

Another study concerned information transport in spatially extended systems. Can localized chaos cause chaos everywhere through a transport process? We find that the transport of information can be detected using the time-delayed mutual information between measurements made at different spatial points [3]. This concept can provide insight into mechanisms that create chaos in spatially extended systems.

Publications

1. "Information and entropy in strange attractor," A. M. Fraser, IEEE Transactions on Information Theory 35, 245-262 (1989).

2. "Reconstructing attractors from scalar time series: a comparison of singular system and redundancy criteria," A. M. Fraser, Physica D 34, 391-404 (1989).

3. "Information transport in spatiotemporal systems," J .A. Vastano and H. L. Swinney, Phys. Rev. Lett. 60, 1773-1776 (1988).

\section{Analysis of strange attractors}

Strange attractors can be characterized in terms of the unstable periodic saddle orbits, which are dense on the attractor. A method has been developed for locating the saddle orbits, and this method has been applied to phase portraits reconstructed from data from experiments on the Belousov-Zhabotinskii reaction [4]. The eigenvalues associated with the saddle orbits are in good accord with the exponent determined by other methods.

Several algorithms have been proposed for determining the dimension of attractors reconstructed from laboratory data. We have compared the Grassberger-Procaccia correlation method with the Badii-Politi nearest neighbor method [5]. These methods yield generalized dimensions $D_{2}$ and $D_{1}$, respectively, but in prastice these two dimensions are usually equal within the experimental uncertainty. The nearest neighbor method is found to be more reliable for attractors with dimension in the range 3-7; neither method is reliable for larger dimensional attractors given by typical data sets.

Publications

4. "The characterization of an experimental strange attractor by periodic orbits," D.P. Lathrop and E.J. Kostelich, Phys. Rev A 40, 4028-4031 (1989).

5. "Practicai considerations in estimating dimension from time series data," E.J. Kostelich and H.L. Swinney, Physica Scripta 40, 436-441 (1989). 


\section{Noise reduction}

Information about the dynamics described by a strange attractor can be used to improve the signal-to-noise ratio of the original time series data for data arising from a low dimensional deterministic process $[6,7]$. An algorithm has been developed to examine the phase space motion of small clusters of points and to identify and correct errors arising from noise. This noise reduction approach is quite different from traditional signal processing techniques because the information used to reduce the noise is not localized in either the time or frequency domain. The method has been applied to chaotic data from laboratory experiments on the Couette-Taylor system and the background noise level in the power spectrum has been reduced by more than a factor of four, thereby uncovering some sharp frequency components previously hidden by noise.

\section{Publications}

6. "Noise reduction in dynamical systems," E. J. Kostelich and J.A. Yorke, Phys. Rev. A 38, 1649-1652 (1988).

7. "Noise reduction: finding the simplest dynamical system consistent with the data," E. J. Kostelich and J.A. Yorke, Physica D 41, 183-196 (1990).

\section{Multifractals}

The Renyi (or "generalized") dimensions, $D_{\mathrm{q}}$, and the spectrum of singularities, $f(\alpha)$, have been very useful in the study of fractal objects and especially of the attractors which arise in dynamical systems. For example, the renormalization group has been used to calculate $f(\alpha)$ for the attractor which exists at the completion of the period doubling sequence [53]. To compare with experiment, one would in principle have to adjust the control parameter exactly to the critical value and measure an infinite number of points corresponding to the infinits period. We have shown that small deviations from the critical value of the control parameter can have a significant effects on the functions $D_{q}$ and $f(\alpha)[8]$. Moreover, certain of these deviations from the ideal behavior depend in a characteristic (universal) way on the properties (e.g., the Feigenbaum numbers) of the ideal attractor. Thus, deviations from the ideal may be used to identify and characterize the period doubling attractor.

We have investigated the scaling properties of multifractal functions near an attractor-repeller transition of a one-dimensional map (a crisis) [9]. The results suggest the existence of universality classes in the scaling of multifractal functions at the onset of a repeller. We have also shown how a small asymmetry in the amplitude of a onedimensional map at its maximum affects the multifractal properties of associated attractors or repellers [10].

Estimates of fractal dimension $D_{0}$ obtained from box-counting algorithms have large errors that arise from the lacunarity of attractors (loosely speaking, lacunarity refers to holes in a fractal attractor) [11]. Thus estimates of $D_{0}$ will inevitably vary widely, depending on the range of scales examined; unfortunately, only a rather narrow range of scales is often available for experimental data.

\section{Publications}

8. "Finite-size effects on the $f(\alpha)$ spectrum of the period-doubling attractor," M. G. Cosenza, W.D. McCormick, and J.B. Swift, Phys. Rev. A 39, 2734-2737 (1989).

9. "Scaling properties of multifractal functions at an attractor-repeller transition," M. G. Cosenza and J. B. Swift, Phys. Rev. A 41, 6615-6617 (1990).

10. "Influence of asymmetry on multifractal properties of maps," M. G. Cosenza and J. B. Swift, Phys. Rev. A 43, 4095-4099 (1991).

11. "Fractal dimensions and $f(\alpha)$ spectrum of the Henon attractor," A. Arneodo, G. Grasseau, and E. J. Kostelich, Phys. Lett. A 124, 426-432 (1987). 


\section{Final Report--page 4}

\section{OSCIILATIONS AND CHAOS IN HOMOGENEOUS CHEMICAL SYSTEMS}

\section{Oscillations}

Experiments over a wide range of concentrations in the manganese-catalyzed Belousov-Zhabotinskii reaction yield long bifurcation sequences involving complex periodic states but, surprisingly, no quasiperiodicity or chaos [54]. The behavior along an arbitrarily chosen path in parameter space appears to be like that expected only for a very special path, the codimension-2 path corresponding to an invariant torus at criticality (where the frequency-locked Arnold tongues have the full measure). An analysis of the theory of circle maps has revealed a way in which the observed behavior can occur along codimension-1 paths in parameter space, and a model yields behavior in good accord with the observations [12].

A variant of the chlorite-iodide-malonic acid reaction has been studied in a closed well-stirred system to understand better the mechanism of this reaction, which is being used in the study of chemical spatial patterns. These experiments revealed previously unobserved long-lived oscillations that could play a role in the spatial patterns observed using this reaction [13].

Another experiment examined the effect of stirring in open and closed reactors containing a variant of the Belousov-Zhabotinskii reaction. No evidence was found for the effects that had been conjectured to arise from micromixing or statistical fluctuations [14].

Publications

12. "Slow manifolds and mixed-mode oscillations in the Belousov-Zhabotinskii reaction," D. Barkley, J. Chem. Phys. 89, 5547-5559 (1988).

13. "Long-lived oscillations in the chlorite-iodide-malonic reaction in batch," Z. Noszticzius, Q. Ouyang, W. D. McCormick and H. L. Swinney, J. Am. Chem. Soc.114, 4290-4295 (1992).

14. "Stirring effects in the $B Z$ reaction with oxalic acid-acetone mixed substrate in a batch reactor and in a CSTK," Z. Noszticzius, W. Horsthemke, W. D. McCormick, and H. L. Swinney, in Spatial Inhomogeneties and Transient Behavior in Chemical Kinetics, ed. by G. Nicolis, P. Gray, F. Baras, P. Borckmans and S. Scott (Manchester University Press, 1990), pp. 647-652.

\section{Chaos}

Despite the many reports in the past decade on chaos in chemical systems, journal articles continue to question whether or not chaos - deterministic nonperiodic behavior - can occur in well-controlled chemical systems. We find that there is an overwhelming body of experimental, numerical, and theoretical evidence for the existence of low-dimensional chaos in nonequilibrium chemical reactions [15]. Recently it was suggested that even though chaos has become well established in experiments at low flow rates in stirred flow reactors, at high flow rates the observed nonperiodic behavior could arise from fluctuations in flow and stirring rates rather than homogeneous chaos [55]. We examined this possibility by repeating and extending the early experiments of Hudson and Mankin [56] on the Belousov-Zhabotinskii reaction at high flow rates. Our observations of a sequence of bifurcations leading to chaos agreed remarkably well (within a few percent) with Hudson and Mankin's observations of a decade earlier, and tests with different perturbants and with imposed fluctuations of the flow rate or stirring rate demonstrated that the high flow rate chaos is quite robust [16]. Moreover, the observations agreed well with a recent model of the reaction. Thus chemical chaos is well established at high flow rates as well as low flow rates.

In an experiment on the Belousov-Zhabotinskii reaction we have observed an abrupt transition that has the character of a crisis [17]. (A crisis is a sudden change in dynamical behavior that occurs when a chaotic attractor collides with an unstable coexisting orbit or its stable manifold.) The interpretation of the observed behavior as a 
crisis was corroborated by a numerical analysis of a seven-variable model of the reaction. The waveforms, attractors, and maps obtained in the simulation were remarkably similar to those obtained in the laboratory experiment.

We have examined the route to chaos through subharmonic intermittency ("Type III intermittency") and have shown that this route to chaos is difficult to distinguish from a subcritical period doubling transition [18]. Necessary criteria for the establishment of a continuous transition were obtained from considerations of one-dimensional maps. Experiments on the Belousov-Zhabotinskii reaction demonstrate that under some conditions this type of intermittency can be definitively observed in experiments.

\section{Publications}

15. "Chemical chaos: from hints to confirmation," F. Argoul, A. Arneodo, P. Richetti, J.C. Roux, and H.L. Swinney, Acc. Chem. Res. 20, 436-442 (1987).

16. "Confirmation of high flow rate chaos in the Belousov-Zhabotinskii reaction," L. Gyorgyi, R. J. Field, Z. Noszticzius, W. D. McCormick and H. L. Swinney, J. Phys. Chem. 96, 1228-1233 (1992).

17. "A crisis in the Belousov-Zhabotinskii reaction: experiment and simulation," P. Richetti, P. De Kepper, J. C. Roux, and H.L. Swinney, J. Stat, Phys. 48, 977-990 (1987).

18. "Experimental demonstration of subtleties in subharmonic intermittency," N. Kreisberg, W. D. McCormick, and H. L. Swinney, Physica D 50, 463-477 (1991).

\section{Bifurcation diagrams as fingerprints}

An attempt in 1987 to repeat some of our early observations of a particular bifurcation sequence leading to chaos in the Belousov-Zhabotinskii reaction led to rather different results to our dismay. Careful detective work finally led to the discovery that for some conditions the reaction is extremely sensitive to certain trace impurities - only a few parts per million of iron impurity can dramatically alter the bifurcation sequence, even completely suppressing chaos [19]. (Tests of malonic acid from six vendors showed that they all contained impurities on the level of ppm; a purification method was developed to remove these impurities.) We then considered the effect of a variety of impurities on the Belousov-Zhabotinskii reaction for a wide range of control parameters and found that bifurcation diagrams can serve as fingerprints of the impurities [20]. By comparing the fingerprints for lifferent perturbants it is possible to gain insight into the mechanism of a reaction.

Publications

19. "Effect of trace impurities on a bifurcation structure in the Belousov-Zhabotinskii reaction and preparation of high-purity malonic acid," Z. Noszticzius, W. D. McCormick, and H. L. Swinney, J. Phys. Chem. 91, 5129-5134 (1987).

20. "Use of bifurcation diagrams as fingerprints of chemical mechanisms," Z. Noszticzius, W.D. McCormick, and H.L. Swinney, J. Phys. Chem. 93, 2796-2800 (1989).

\section{SPATIOTEMPORAL PATTERNS IN REACTION-DIFFUSION SYSTEMS}

A major goal during the current grant period has been to develop reactors suitable for the study of sustained chemical spatial patterns. Without such a device it is not possible to study bifurcations in reaction-diffusion systems. Past experiments on the formation of chemical structures were conducted in closed (batch) systems such as petri dishes, not in open chemostats such as the stirred tanks used in the study of homogeneous temporal structures [12-20]. The circular and spiral patterns that have been studied in such closed systems evolved inexorably towards thermodynamic equilibrium and decayed as the reagents were consumed.

The first reactor developed to study sustained patterns was the Couette reactor, which uses a hydrodynamic structure - turbulent Taylor vortices - as a quasi-onedimensional system in which sustained patterns can be obtained [21-24]. 


\section{Final Report--page 6}

Another approach to the study of sustained chemical patterns uses a chemically inert gel that is in contact with continuously refreshed reservoirs at one or more boundaries. The gel prevents convection; hence patterns arise solely from the interaction of reaction and diffusion. We have used these gel reactors to study chemical waves [25-29], stationary ("Turing") patterns [30-35], and spatiotemporal chaos [36]. These simple gel reactors have been duplicated in several laboratories in the U.S. and Europe and have led to discoveries of a variety of chemical patterns in our laboratory and elsewhere.

\section{The Couette reactor: a one-dimensional reaction-diffusion system}

The Couette reactor makes novel use of the circular Couette system, which consists of a pair of concentric cylinders, the inner one of which rotates in our system. Chemical reagents are fed and removed at each end of the annulus, but the flow rates are carefully adjusted so that there is no net axial mass flux. For sufficiently rapid rotation rates of the inner cylinder, the Taylor vortices that encircle the inner cylinder are turbulent, and their only role in the chemical system is to enhance the transport. The reagents, which are passive scalars, are well mixed in the radial and azimuthal directions on a time scale short compared to the characteristic time for the diffusive transport in the axial direction [57]. Thus the Couette reactor is effectively onedimensional reaction-diffusion system. The effective diffusion coefficient, which is the same for all species (typically $0.1 \mathrm{~cm}^{2} / \mathrm{s}$ ), can be varied by varying the rotation rate of the inner cylinder.

The Couette reactor has been used in the first observations of a sequence of spatiotemporal regimes in a laboratory reaction-diffusion system [21]. The following sequence of regimes is observed as the gradient in oxidizer concentration of the Belousov-Zhabotinskii reaction is increased with other control parameters held fixed: steady, periodic, quasiperiodic, frequency-locked, period-doubled, and chaotic. This sequence of bifurcations in spatiotemporal patterns is described well by a simple reaction-diffusion model with only two species. The general stability of fronts in a Couette reactor has also been considered and conditions have been found under which oscillatory fronts become chaotic [22-23].

Another experiment on the Couette reactor demonstrates that this system can serve as a new tool for enhancing the recovery of short-lived intermediate species in a reaction [24]. The experiment was conducted on an arsenite-iodate reaction, and the short-lived intermediate was triiiodide. The observations agree well with the predictions of a onedimensional model that has no adjustable parameters.

\section{Publications}

21. "Bifurcation to spatially induced chaos in a reaction-diffusion system," J. A. Vastano, T. Russo and H. L. Swinney, Physica D 46, 23-42 (1990).

22. "Modeling reaction-diffusion pattern formation in the Couette reactor," J. Elezgaray, A. Arneodo, J. Chem. Phys. 85, 323-350 (1991).

23. "Instabilities in reaction-diffusion systems," A. Arneodo, J. Elezgaray, J. Pearson, T. Russo, Physica D 49, 141-160 (1991).

24. "Spatial variation of a short-lived intermediate chemical species in a Couette reactor," R. D. Vigil, Q. Ouyang, and H. L. Swinney, J. Chem. Phys. 96, 6126-6131 (1992).

\section{Spirals and waves}

The first experiment to yield sustained chemical waves was conducted in a thin annular gel layer in contact with reservoirs at the inner and outer edges of the gel. Rotating waves - pinwheels - were observed in the Belousov-Zhabotinskii reaction for a wide range in parameters [25-26]. A numerical simulation of the two-species TysonFife reaction-diffusion model yielded a wave form in qualitative agreement with that 
observed in laboratory experiments [27]. With further variation in parameters, a symmetry-breaking transition was observed from a wave with $m$-fold rotational symmetry to one with $\mathrm{m} / 2$ rotational symmetry [28].

Transient chemical spirals have been studied in experiments on closed systems for more than two decades. It was observed that in some cases the tip of the decaying spiral seemed to move approximately in a circular path while in other cases it "meandered" [58]. Using an open system, a continuously fed gel reactor, we have made the first laboratory study of spirals that can be maintained indefinitely. A transition is observed from the uniform (nonpatterned) state to spirals that rotate periodically, and, with further variation of feed chemical concentrations, there is a bifurcation from periodic to quasiperiodic rotation of the spiral tip [29]. The observation of a supercritical secondary bifurcation to quasiperiodic spiral motion has motivated several theoretical analyses of reaction-diffusion models; these analyses [e.g., see 59 and 60] are in accord with our observations.

Publications

25. "Sustained chemical waves in an annular gel reactor: a chemical pinwheel," $Z$. Noszticzius, W. Horsthemke, W. D. McCormick, H. L. Swinney, and W. Y. Tam, Nature 329, 619-621 (1987).

26. "Sustained chemical waves in a Turing-Nicolis-Prigogine ring reactor," $Z$. Noszticzius, W. Horsthemke, W. D. McCormick, and H. L. Swinney, in Spatial Inhomogeneties and Transient Behavior in Chemical Kinetics, ed. by G. Nicolis, P. Gray, F. Baras, P. Borckmans and S. Scott (Manchester University Press, 1990), pp. 429-432.

27. "Semi-implicit solution of a reaction-diffusion system with stiff kinetics," D. A. Vasquez, to appear, J. Comr. Chem., 1992.

28. "Symmetry breaking in a chemical pinwheel," N. Kreisberg, W. D. McCormick, and H. L. Swinney, J. Chem. Phys. 91, 6532-6533 (1989).

29. "Periodic to quasiperiodic transition of chemical spiral rotation," G. Skinner and H. L. Swinney, Physica D 48, 1-16 (1991).

\section{Turing patterns}

Turing's classic 1952 paper [61] entitled "The Chemical Basis of Morphogenesis" has been the basis for much theoretical work on pattern formation in biological systems [see, e.g., 62 and 63]. However, as we noted in 1988 [ref. 30, p. 6181]:

"There has not been any corroboration of Turing's well-known theory of stationary pattern formation in chemical reaction-diffusion systems. We suggest that the use of continuously fed unstirred reactors should make it possible to observe Turing bifurcations in laboratory experiments. The observation of a steady chemical pattern in such a reactor would not by itself qualify as a convincing demonstration of a Turing pattern. It is necessary to observe the destabilization of a homogeneous steady state as some parameter is increased."

Subsequently we made the first observation of a Turing bifurcation: a nonhysteretic transition from a homogeneous steady state to a stationary two-dimensional hexagonal pattern of concentrations, as pictured in Fig. 1(a) and 1(c) [31]. Patterns were observed to emerge spontaneously from the homogeneous state in a chlorite-iodide-malonic acid reaction; this reaction had been used earlier by De Kepper and co-workers in their discovery of quasi-one-dimensional stationary patterns [64]. With further change in parameter we observed a secondary transition from the hexagonal pattern to a striped pattern, as shown in Fig. 1(b).

Amplitude equation analyses of pattern formation in two-dimensional systems generally predict that, in the absence of special symmetries, a supercritical primary bifurcation from the uniform state should lead to a hexagonal pattern [65]; this was the case in the first observation of a Turing bifurcation [31]. Subsequent observations for 

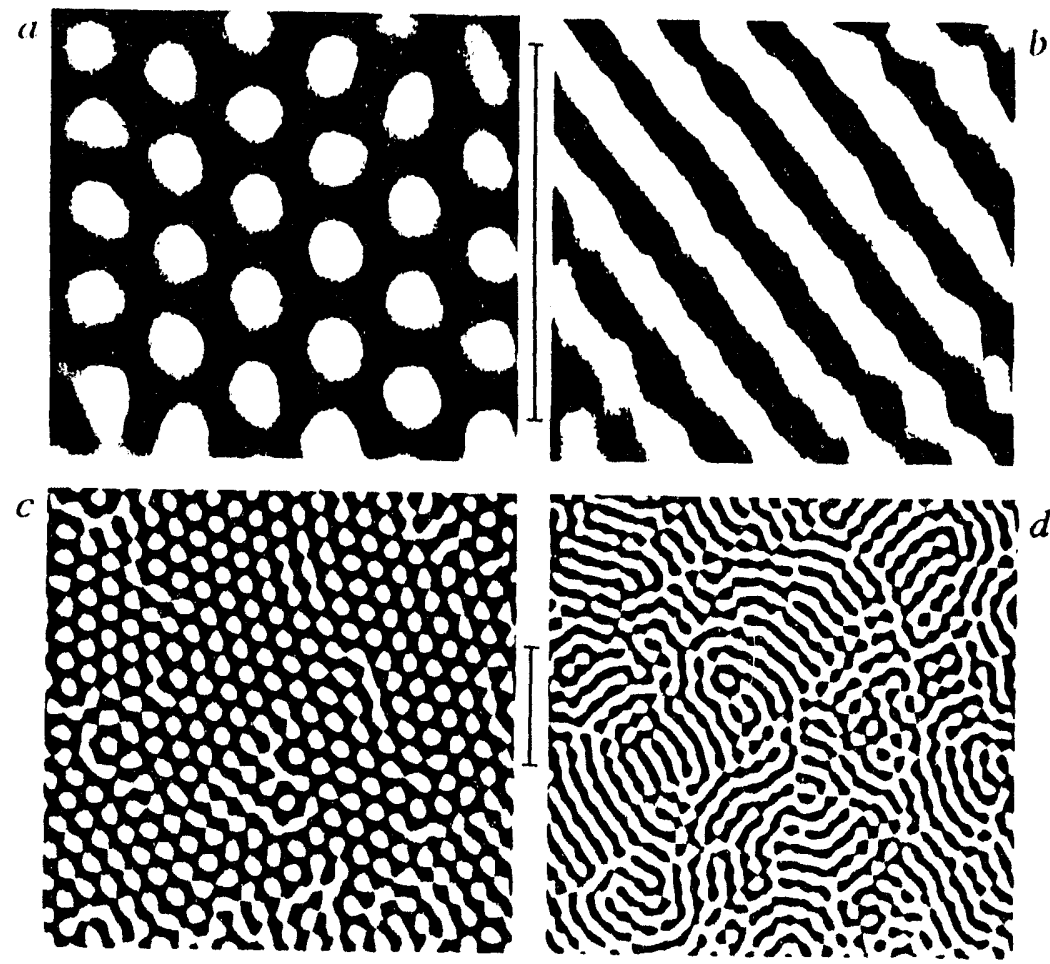

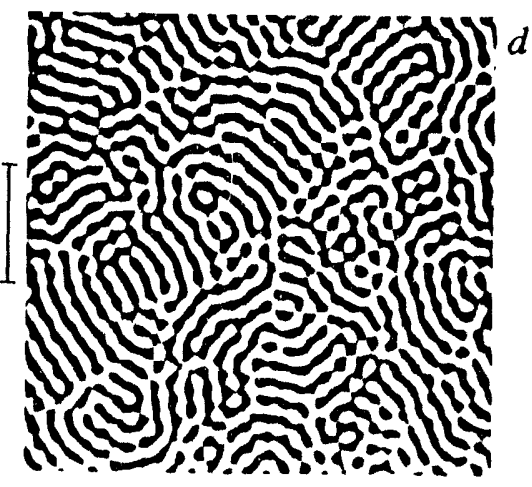

FIG. 1. Stationary reactiondiffusion (Turing) patterns formed in a continuously fed laboratory gel reactor (from [31]): (a) and (c) hexagonal patterns; (a) is an enlargement of part of (c); (b) a striped pattern; (d) a striped pattern for conditions near the onset of time-dependent disordered ("turbulent") patterns. The wavelength in each pattern is approximately $0.2 \mathrm{~mm}$; only small parts of the $25 \mathrm{~mm}$ diameter reactor are shown the bars in the center represent $1 \mathrm{~mm}$.

another range in control parameters have revealed a transition from a uniform state directly to a striped pattern, and within the experimental resolution this transition also is supercritical [32]. A secondary transition then leads from the stripes to hexagons; the latter transition is hysteretic - there is a control parameter range in which both stripes and hexagons are stable.

The Turing patterns in [31] and [64] were observed using starch as an indicator. To clarify the role of starch we conducted experiments without starch and obtained sustained patterns [33] very similar to those obtained previously using starch. The patterns obtained without starch are rendered visible primarily by the variations in the gel refractive index, presumably due to nonuniform swelling of the gel.

The experiments on Turing patterns in our laboratory and elsewhere were all been conducted using the chlorite-iodide-malonic acid (CIMA) reaction with Thiodene from Prolabo as an indicator. We investigated two other indicators, soluble starch and polyvinyl alcohol, and found that they yielded patterns similar to those obtained with Thiodene [34]. In addition, we found that Thiodene is not simply a soluble starch, as had been assumed, but is made by mixing $7 \%$ starch with $93 \%$ molten urea.

The observed sustained stationary and spiral wave patterns were obtained in several different types of gel reactors [25, 28-29, 30-34], each of which uses a membrane or porous glass (Vycor) or a glass capillary array to separate the gel from continuously refreshed chemical reservoirs. Recently we have found that patterns can form in a thin gel in direct contact with a stirred reservoir [35]. This extremely simple reactor is easy to construct and hence should serve as the basic tool for many future studies of chemical patterns.

\section{Publications}

30. "Turing patterns in an open reactor," J. A. Vastano, J. E. Pearson, W. Horsthemke, and H. L. Swinney, J. Chem. Phys. 88, 6175-6181 (1988). 
31. "Transition from a uniform state to hexagonal and striped Turing patterns," Q. Ouyang and H. L. Swinney, Nature 352, 610-612 (1991).

32. "Spatial bistability of two-dimensional Turing patterns in a reaction-diffusion system," $Q$. Ouyang, Z. Noszticzius, and H. L. Swinney, J. Chem. Phys. 96, 6773-6776 (1992).

33. "Turing patterns visualized by index of refraction variations," K. J. Lee, W. D. McCormick, Z. Noszticzius, and H. L. Swinney, J. Chem. Phys. 86, 4048-4049 (1992).

34. "The effect of Turing pattern indicators on CIMA oscillators," Z. Noszticzius, Q. Ouyang, W. D. McCormick, and H. L. Swinney, J. Phys. Chem. 96, 6302-6307 (1992).

35. "Turing patterns in a simple gel reactor," R. D. Vigil, Q. Ouyang, and H. L. Swinney, Physica A 188, 17-25 (1992).

\section{Chemical turbulence}

For conditions beyond those corresponding to the emergence of stationary patterns we have observed a transition to a disordered time-dependent pattern, which we have called chemical turbulence [36]. The transition to time dependence is accompanied by a large increase in the number of defects, which suggests that this is an example of defect-mediated turbulence.

Publication

36. "Transition to chemical turbulence," Q. Ouyang and H. L. Swinney, CHAOS Journal 1, 411-420 (1991).

\section{Reviews}

Our observations of sustained chemical spatial patterns are summarized in three conference proceedings publications [37-39].

Publications

37. "Temporal and spatial patterns in chemical systems," H. L. Swinney, W. Horsthemke, W. D. McCormick, Z. Noszticzius, and W. Y. Tam, in Dynamic Patterns in Complex Systems, ed. by J. A. Kelso, A. J. Mandell, and M. F. Shlesinger (World Scientific, Singapore, 1988), pp. 112-120.

38. "Experiments on temporal and spatial chemical patterns," H. L. Swinney, W. D. McCormick, and Z. Noszticzius, Reaction Kinetics and Catalysis Letters 42, 253-262 (1990).

39. "Spatiotemporal patterns in reaction-diffusion systems," H. L. Swinney, N. Kreisberg, W. D. McCormick, Z. Noszticzius, and G. Skinner, in CHAOS, Soviet-American Perspectives in Nonlinear Science, ed. by D. K. Campbell (American Institute of Physics, New York, 1990), pp. 197204.

\section{INSTABIITIES AND PATTERNS IN FLUID DYNAMICS}

\section{Modulated Rayleigh-Bénard convection and Taylor-Couette flow}

One of the principal reasons for studying modulated Rayleigh-Bénard convection is that it provides an interesting system for investigating issues in pattern selection and competition, both experimentally and theoretically. If the temperature of the bounding horizontal plates is static and uniform in space, then, in the Oberbeck-Boussinesq approximation the pattern that appears immediately above the onset of convection is in the form of rolls. By means of a weakly nonlinear theory, Roppo, Davis and Rosenblat [66] predicted that if the temperature is uniform but modulated in time, the initial pattern should be in the form of hexagons. We have used a model [67] that goes beyond weakly nonlinear theory to describe the hexagonal pattern near onset in modulated convection and to describe the competition between hexagonal and roll patterns at higher values of the average Rayleigh number. This model is particularly well suited to guiding experimenters in their search in a rather large parameter space (particularly the frequency and amplitude of the modulation) for the range of existence of the 
hexagonal pattern. Our predicted stability limits for hexagons and rolls agree well with experiments conducted by Meyers, Cannell, and Ahlers at UC Santa Barbara [40]. More general time-dependent boundary conditions, such as sinusoidal modulation of the top and bottom plates with various phase differences, have also been considered [41], but no more favorable conditions were found for the existence of hexagons than the simple modulation of one plate considered in [40]. At high modulation frequency, a spatial scaling based a thermal Stokes layer, whose thickness decreases as the inverse square root of the frequency, rather than a scale based on the depth of the fluid layer is appropriate [42]; the predictions of this analysis compare well with an experiment conducted by Niemela and Donnelly at the University of Oregon [68].

We have also studied the effect of modulation on the onset of secondary flow in the Taylor-Couette system [43], and the predictions were in qualitative agreement with experiments by Walsh and Donnelly [69].

Publications

40. "Pattern competition in temporally modulated Rayleigh-Bénard convection," C. W. Meyer, D. S. Cannell, G. Ahlers, J. B. Swift, and P. C. Hohenberg, Phys. Rev. Lett. 61, 947-950 (1988).

41. "Rayleigh-Bénard convection with time-dependent boundary conditions," J. B. Swift and P. C. Hohenberg, Phys. Rev. A 39, 4132-4136 (1989).

42. "Modulated convection at high frequencies and large modulation amplitudes," J. B. Swift and P. C. Hohenberg, Phys. Rev. A 36, 4870-4875 (1987).

43. "Onset of secondary flow in the modulated Taylor-Couette system," X. Wu and J. B. Swift, Phys. Rev. A 40, 7197-7201 (1989).

\section{Effects of noise near the onset of Rayleigh-Bénand convection}

Ordinarily, macroscopic fluid flow such as Rayleigh-Bénard convection can be discussed in terms of deterministic equations such as the Navier-Stokes equation, and the effects of the ever present thermal noise can be neglected. However, certain experiments [70] appear to require stochastic effects for their physical interpretation. We have examined a stochastic Landau equation and have obtained an approximate analytic expression for the probability distribution for the case in which the bifurcation parameter is time dependent [44]. This expression was used in [70] to extract the strength of the noise present in an experiment in which the Rayleigh number was ramped through onset as time increased. The measured value of the noise was then used to explain quantitatively certain aspects of an experiment in which the Rayleigh number was modulated sinusoidally in time [45].

Publications

44. "Stochastic Landau equation with time-dependent drift," J. B. Swift, P. C. Hohenberg, and G. Ahlers, Phys. Rev. A 43, 6572-6580 (1991).

45. "Comment on "Initial stages of pattern formation in Rayleigh-Bénard convection," J. B. Swift and P. C. Hohenberg, Phys. Rev. Lett. 60, 75 (1988).

\section{ELECTRODEPOSITION CLUSTERS}

Experiments have been conducted on the fractal properties of zinc aggregates generated by electrodeposition in a system consisting of two parallel zinc electrodes, 0.1 $\mathrm{mm}$ in diameter, separated by a distance of $50 \mathrm{~mm}[46,47]$. Clusters were examined in the asymptotic limit of low voltage and low zinc sulfate concentration. The clusters were found to be self-similar and to have a generalized dimension $\mathrm{D}_{\mathrm{q}}=1.66 \pm 0.08$, independent of $q$. Small mass clusters were also generated in a numerical simulation of diffusion limited aggregation (DLA) in a strip geometry. Clusters with 3,000 to 30,000 
particles were found to be self-similar, with $D_{q}=1.60 \pm 0.02$. These results suggest that electrodeposition and diffusion-limited clusters have a similar geometrical structure.

The anode-cathode potential was found to oscillate in some experiments conducted with constant current $[48,49]$. Initially the oscillations are essentially periodic. As the growth progresses, a transient period doubling cascade leading to cha0s is observed [48, 49]. Analysis of video images reveals that the oscillations in growth rate are in phase with the oscillations in potential, and, surprisingly, are in phase across the entire cell; such long range coherence cannot be described by a diffusive process such as DLA.

Publications

46. "Self-similarity of diffusion-limited aggregates and olectrodeposition clusters," F. Argoul, A. Arneodo, G. Grasseau, and H. L. Swinney, Phys. Rev. Lett. 61, 2558-2581 (1988).

47. "Reply to comment on self-similarity of DLA and electrodeposition clusters," F. Argoul, A. Arneodo, G. Grasseau, and H. L. Swinney, Phys. Rev. Lett. 63, 1323 (1989).

48. "Experimental evidence for spatio-temporal chaos in diffusion-limited growth processes," F. Argoul, A. Arneodo, J. Elezgaray and H. L. Swinney, in Nonlinear Phenomena in Growth and Form, ed. by M. BenAmar, P. Pelcé, and P. Tabeling (Plenum Publishing, New York, 1990).

49. "Experimental evidence for homoclinic chaos in an electrochemical growth process," F. Argoul, J. Huth, P. Merzeau, A. Arneodo and H. L. Swinney, Physica D 62, 170-185 (1993).

\section{PATTERNS IN A BALLAST RESISTOR}

Pattern formation was studied experimentally and theoretically for a ballast resistor - a current-carrying ferromagnetic wire at the center of a hydrogen-filled cylinder [50]. The observed patterns were in qualitative accord with theory, but a detailed quantitative comparison of theory and experiment was not possible because of several experimental difficulties, including particularly hydrogen embrittlement of the iron.

Publication

50. "The ballast resistor: an experimental study of a spatial pattern forming system," N. Bujanos, J. Pearson, W. D. McCormick, and W. Horsthemke, Phys. Lett. A 127, 138-142 (1988).

\section{CRACK PROPAGATION}

The support of this grant has enabled us to begin to explore another problem in pattern formation: the pattern formed by a propagating crack in an amorphous material $[50,51]$. Cracks in brittle materials have terminal velocities far below theoretical predictions. To examine this problem we are crack propagation in a brittle plastic (PMMA - polymethylmethacrylate). Velocity measurements with resolution an order of magnitude better than previous experiments reveal a transition at a critical velocity $(330 \pm 30 \mathrm{~m} / \mathrm{s})$. At this transition the velocity begins to oscillate, the mean acceleration drops sharply, and a periodic spatial pattern is formed on the fracture surface. These observations suggest that the dynamics of cracks may be governed by a dynamical instability.

These initial exploratory experiments, which were proposed and led by a colleague in the Center for Nonlinear Dynamics, Professor Michael Marder, indicate that concepts from nonlinear dynamics may prove fruitful in the study of cracks. Therefore, Professor Marder will seek separate funding to establish a theoretical and experimental research program on cracks.

Publications

50. "An instability in dynamic fracture," J. Fineberg, S. Gross, M. Marder, and H. L. Swinney, Phys.

Rev. Lett. 67, 457-460 (1991). 
51. "An instability in the propagation of fast cracks," J. Fineberg, S. P. Gross, M. Marder, and H. L. Swinney, Phys. Rev. B 45-II, 5146-5154 (1992).

\section{ACKNOWLEDGMIENTS}

Investigators Swinney and Swift acknowledge the many contributions of students, postdocs, faculty colleagues and visitors to this research program. We would also like to acknowledge stimulating collaborations with the following scientists at other institutions: Z. Noszticzius (Technical University of Budapest), who was a visitor in our laboratory for nearly two years of the current grant period; A. Arneodo, F. Argoul, P. De Kepper, and J.C. Roux (Centre de Recherche Paul Pascal, Bordeaux); P. C. Hohenberg (AT\&T Bell Laboratories); G. Ahlers and D. S. Cannell (UC Santa Barbara); J. A. Yorke (U. Maryland); and L. Gyorgyi and R. J. Field (U. Montana).

\section{REFWRINCESTO OTHIGR WORK}

53. D. Bensimon, M.H. Jensen, and L.P. Kadanoff, Phys. Rev. A 33, 3622 (1986).

54. J. Maselko and H. L. Swinney, J. Chem. Phys. 85, 6430 (1986).

55. A. Munster and F. W. Schneider, J. Phys. Chem. 95, 2130 (1991).

56. J. L. Hudson and J. C. Mankin, J. Chem. Phys. 74, 6171 (1981).

57. W. Y. Tam and H. L. Swinney, Phys. Rev. A 36, 1374 (1987).

58. A. T. Winfree, Science 175, 634 (1972).

59. D. Barkley, M. Kness, L. S. Tuckerman, Phys. Rev. A 42, 2489 (1990).

60. A. Karma, Phys. Rev. Lett. 65, 2824 (1990).

61. A. M. Turing, Phil. Trans. Roy. Soc. London B 327, 37 (1952).

62. H. Meinhardt, Models of Biological Pattern Formation (Academic, New York, 1982).

63. J. D. Murray, Mathematical Biology (Springer, Berlin, 1989).

64. V. Castets, J. Boissonade, E. Dulos, and P. De Kepper, Phys. Rev. Lett. 64, 2953 (1990).

65. B. Malomed and M. I. Tribel'skii, Sov. Phys. JETP 65, 3013 (1987).

66. M. N. Roppo, S. H. Davis, and S. Rosenblat, Phys. Fluids 27, 796 (1984).

67. P. C. Hohenberg and J. B. Swift, Phys. Rev. A 35, 3855 (1987).

68. J. J. Niemela and R. J. Donnelly, Phys. Rev. Lett. 57, 583 (1986).

69. T. J. Walsh and R. J. Donnelly, Phys. Rev. Lett. 60, 700 (1988).

70. C. W. Meyer, G. Ahlers, and D. S. Cannell, Phys. Rev. A 44, 2514 (1991).

\section{DISCLAIMER}

This report was prepared as an account of work sponsored by an agency of the United States Government. Neither the United States Government nor any agency thereof, nor any of their employees, makes any warranty, express or implied, or assumes any legal liability or responsibility for the accuracy, completeness, or usefulness of any information, apparatus, product, or process disclosed, or represents that its use would not infringe privately owned rights. Reference herein to any specific commercial product, process, or service by trade name, trademark, encestitute or imply its endorsement, recommanufacturer, or avoring by the United States Government or any agency thereof. The views mendation, or favoring by the United States Government or any agency thereof. The villy and opinions of authors expressed herein do 

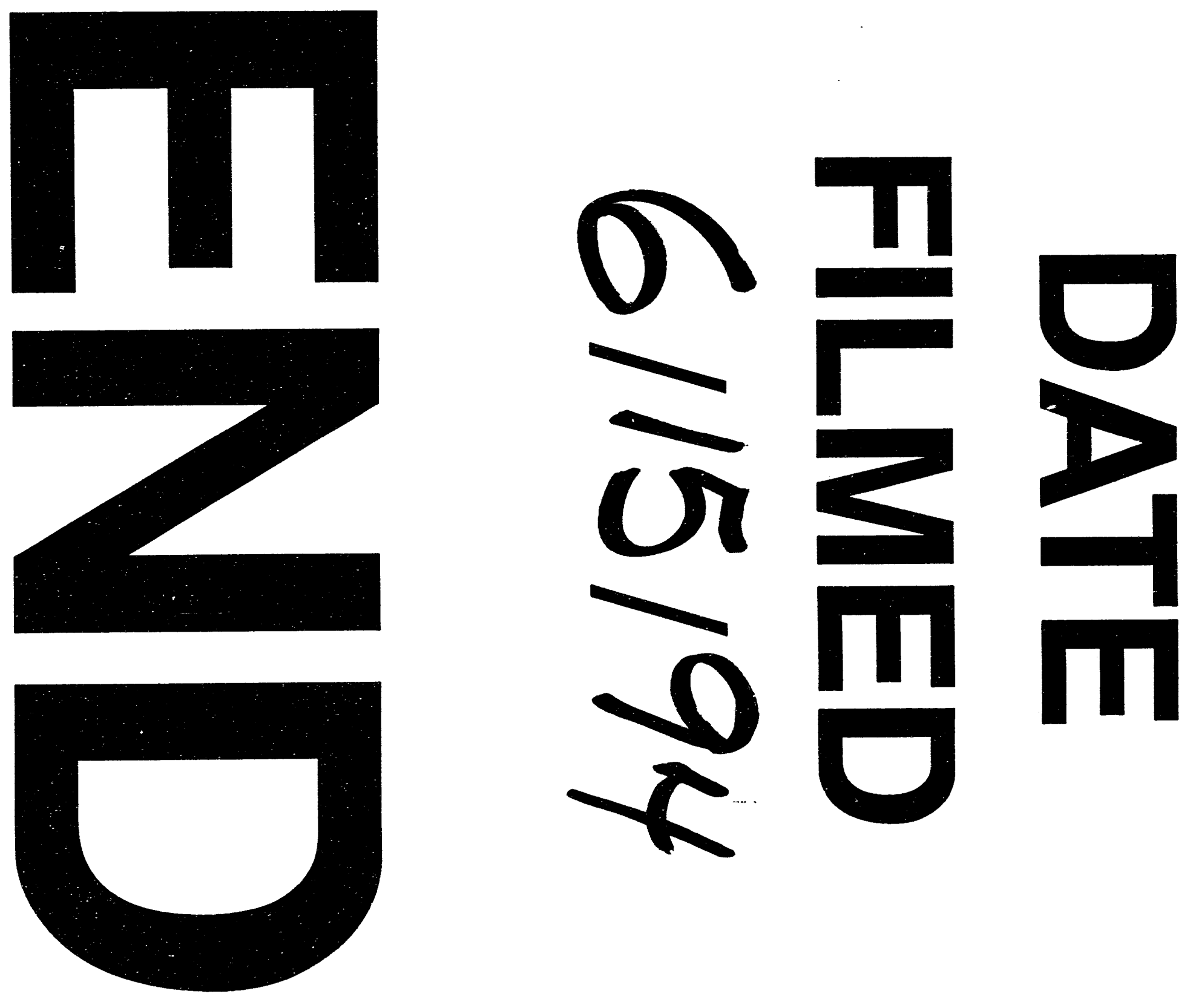
